\title{
A multi-depot location routing problem to reduce the differences between the vehicles' traveled distances; a comparative study of heuristics
}

\author{
Hengameh Hadian $^{\mathrm{a}^{*}}$, Amir-Mohammad Golmohammadi ${ }^{\mathrm{b}}$, Akbar Hemmati $^{\mathrm{c}}$ and Omolbanin Mashkani ${ }^{\mathrm{d}}$
}

${ }^{a}$ Department of Industrial Engineering, University of Nahavand, Nahavand, Iran

${ }^{b}$ Department of Industrial Engineering, Yazd University, Yazd, Iran

${ }^{c}$ Department of Industrial Engineering, K. N. Toosi University of Technology, Tehran, Iran,

${ }^{d}$ University of Technology Sydney, Sydney, Australia

\section{H R O N I C L E}

Article history:

Received February 2, 2018

Accepted June 72018

Available online

June 72018

Keywords:

Location routing problem (LRP)

Vehicle routing; Facility location

Imperialist competitive algorithm

(ICA)

NSGA-II

\begin{abstract}
A B S T R A C T
This paper presents a model to solve the multi-objective location-routing problem with capacitated vehicles. The main purposes of the model are to find the optimal number and location of depots, the optimal number of vehicles, and the best allocation of customers to distribution centers and to the vehicles. In addition, the model seeks to optimize vehicle routes and sequence to serve the customers. The proposed model considers vehicles' traveled distances, service time and waiting time while guaranteeing that the sum of these parameters is lower than a predetermined value. Two objective functions are investigated. First objective function minimizes the total cost of the system and the second one minimizes the gap between the vehicles' traveled distances. To solve the problem, a Multi-Objective Imperialist Competitive Algorithm (MOICA) is developed. The efficiency of the MOICA is demonstrated via comparing with a famous meta-heuristics, named Non-Dominated Sorting Genetic Algorithm-II (NSGA-II). Based on response surface methodology, for each algorithm, several crossover and mutation strategies are adjusted. The results, in terms of two well-known comparison metrics, indicate that the proposed MOICA outperforms NSGA-II especially in large sized problems.
\end{abstract}

(C) 2019 by the authors; licensee Growing Science, Canada

\section{Introduction}

In distribution network design, locating manufacturing facilities and planning distribution routes are two critical issues which are usually tackled separately due to the complexity of the overall problem. However, research has demonstrated that this strategy often leads to highly suboptimal solutions (Salhi $\&$ Rand, 1989). The problem of location-routing overcomes this drawback via integrating the two fundamental problems of facility locating and vehicle routing. This integrated approach has been found to be useful and affordable in different real-life aspects. For example, the distribution of perishable food products (Govindan et al., 2014), blood bank location (Or \& Pierskalla, 1979), waste collection (Caballero et al., 2007), parcel delivery (Wasner \& Zäpfel, 2004), hub location and routing (Çetiner et al., 2010), and mission planning in space exploration (Ahn et al., 2012).

\footnotetext{
* Corresponding author

E-mail address: hengameh.hadian@gmail.com (H. Hadian) 
The common objective for LRPs is to minimize the overall cost of the system. Despite the fact that most real-world LRPs are characterized by more than one conflicting objective, a few papers investigated the problem with different or multiple objective functions (Nagy \& Salhi, 2007). Hence, it is worth studying LRPs dealing with several monetary and non-monetary objective functions. In this paper, a multi-objective LRP with capacitated and homogeneous vehicle fleet is modeled. The main purposes of the model are to find the optimal number and location of depots, the optimal number of vehicles, and the best allocation of customers to distribution centers and to the vehicles. Also, the model tries to optimize vehicle routes and sequence to serve the customers considering their limited capacities. The proposed LRP model deals with optimizing both monetary and non-monetary objective functions at the same time. The monetary objective function seeks to minimize the total cost of the system including the summation of fixed cost of open depots and transportation's variable costs. The nonmonetary objective function minimizes the difference between vehicles' traveled distances. This objective function aims to provide a better trade-off between two problems of facility location and vehicle routing problem. Hence, it is useful to find the optimum routes of vehicles and optimum sequence to serve customers. The given problem is then solved by a new Multi-Objective Imperialist Competitive Algorithm, called MOICA. The proposed meta-heuristic is compared with a well-known evolutionary meta-heuristics, named NSGA II, in terms of two multi-objective comparison metrics for numerous test problems.

The above mentioned properties along with the other classic constraints of the LRPs about distribution centers and sub-tour elimination, and also considering some other graph based constraints form a comprehensive modeling structure. Considering these assumptions and complexities simulatneously make the problem more ralistic, and to the best of our knowledge, have never been discussed in the related literature.

The remining of this paper is organized as follows: Section 2 discusses the contributions of the paper, while in Section 3 problem definition and mathematical model are presented. Section 4 proposes metaheuristics and solution approaches (i.e., MOICA and NSGA II) in addition to reporting parameter setting, comparison metrics, generating numerical problems, and computational results. Finally, conclusion and suggestion for further works are given in Section 5.

\section{Contributions}

\subsection{Contributions in variants of LRPS}

A survey on LRP literature revealed that the research on LRP is quite limited in comparison with vehicle routing problem or location problem (Zarandi et al., 2011). The conceptual foundation of researches on LRP dates back to 1960s (Boventer, 1961; Christofides \& Eilon, 1969; Maranzana, 1964; Watson-Gandy \& Dohrn, 1973; Webb, 1968). The primary studies have identified the close interface among location and transportation problems. However, these studies were far from capturing the total complexity of the LRP. Salhi and Rand (1989) demonstrated that making separate decisions for location and routing problems may lead to highly suboptimal solutions, even if the location decisions must be made for a long term (Salhi \& Nagy, 1999). During the past decades, several review papers have been published on LRP literature (Balakrishnan et al., 1987; Min et al., 1998; Nagy \& Salhi, 2007). Furthermore, very recent surveys by Prodhon and Prins (2014) and Drexl and Schneider (2015) represented a classification of LRP variants and discussed recent developments in the field. In this paper, a novel model of multi-objective LRP is proposed paying more attention to the recent developments.

\subsection{Contributions in objective function}

The common objective for LRPs is to minimize the total cost of the system. As it was mentioned before, although most real-case LRPs are characterized by more than one conflicting objectives, just a few 
papers investigated a different objective or considered multiple objective LRPs (Nagy \& Salhi, 2007). However, some recent papers have dealt with several monetary and non-monetary objective functions at the same time. Tavakkoli-Moghaddam et al. (2010) proposed an integrated mathematical model for a bi-objective LRP with optional customers. The monetary objective is to minimize the total cost of the system, while the non-monetary objective is to maximize the total customer demand served. The authors applied two meta-heuristics to solve the problem, named Multi-Objective Scatter Search and Elite TS (Gu et al., 2007). Wang et al. (2014) developed a nonlinear integer open LRP for relief distribution problem where optimization of the total cost, travel time, and reliability with split delivery are considered simultaneously. The authors used the non-dominated sorting genetic algorithm and nondominated sorting differential evolution algorithm to solve the problem. Martínez-Salazar et al. (2014) proposed a non-linear bi-objective two echelon LRP with several capacitated facilities and fixed opening costs on both levels. The first objective is to minimize the total distribution cost and the second is to balance route duration. The authors offered two meta-heuristic solution algorithms: a scatter tabu search procedure and a Non-dominated Sorting GAII (NSGA-II).

In this paper, the proposed LRP model copes with optimizing two monetary and non-monetary objective functions. The monetary objective function is to minimize the total cost of the system and is defined as a summation of fixed cost of open depots and the transportation's variable costs. The nonmonetary objective function is to minimize the differences between vehicles' traveled distances to provide a better trade-off between two problems of facility location and vehicle routing problems. Consequently, the non-monetary objective function is useful to find the optimum routes of vehicles and optimum sequence to serve customers. To the best of our knowledge, investigating all abovementioned goals is unique in the literature and can be considered as an important contribution of the paper.

\subsection{Contributions in solution approaches and meta-heuristics}

The LRPs combine two essential sub-problems of location-allocation and vehicle routing problems. Since both of these problems are NP-hard (Megiddo \& Supowit, 1984; Salhi \& Nagy, 1999), obviously the location-routing problem is also NP-hard. In such complicated combinatorial problems, exact solutions and optimization solvers such as LINGO, GAMS and CPLEX are inefficient, especially on large-sized problems (Diabat, 2014; Roozbeh Nia et al., 2015). Hence, to solve the large size instances of the problem, meta-heuristic search algorithms are needed to obtain near optimal solutions in acceptable computing time. Many studies have successfully employed several meta-heuristic approaches to solve various optimization models of LRP. Some of these meta-heuristic algorithms are: scatter search (Tavakkoli-Moghaddam et al., 2010), simulating annealing (Mohammadkhanloo \& Bashiri, 2013; Yu \& Lin, 2015), tabu search (Fakhrzada \& Esfahanib, 2013; Goścień et al., 2015), particle swarm optimization (Marinakis et al., 2013; Norouzi et al., 2015), genetic algorithm (Karakatič \& Podgorelec, 2015; Setak et al., 2014), evolutionary algorithm (Koç et al., 2015; Prodhon, 2011), neural networks (Kopfer et al., 2005; Schwardt \& Fischer, 2008) and ant colony optimization (Sim \& Sun, 2003; Ting \& Chen, 2013). Among these approaches, the population-based algorithms are usually preferred to others and in most cases show better performances (Roozbeh Nia et al., 2015).

An evolutionary algorithm inspired by imperialist competition process, named Imperialist Competitive Algorithm (ICA), was introduced by Atashpaz-Gargari \& Lucas (2007). This evolutionary algorithm shows some attractive features to be employed in solving LRPs. As the most important feature, ICA searching process is designed well. The probability of falling into the trap of local optima, especially in problems with very large search spaces, is efficiently reduced using different well-designed operators such as relocation of solutions in the search space frequently (Jula et al., 2015). In addition, the ICA provides extensive areas of innovation because it is younger than most proposed evolutionary algorithms (Jula et al., 2015). It should be noted that the ICA has presented better performance compared with most population based algorithms in terms of both the objective function values and computational time (Roozbeh Nia et al., 2015). Shiripour et al. (2012) proposed two meta-heuristics, 
namely GA and ICA, to solve a multi-facility location problem. They showed that the proposed ICA has better performance from both the computing time and solution accuracy point of views in comparison with GA. Mohammadi-Ivatloo et al. (2012) used ICA for solving non-convex dynamic economic power dispatch problem. The authors compared the results of this algorithm with those of particle swarm optimization (PSO) and the best known genetic algorithm (GA), and as a consequence the ICA has more efficient results. In this paper, a novel meta-heuristic algorithm, which is called Multi-Objective Imperialist Competitive Algorithm (MOICA), is developed to solve the problem exploiting the ICA properties.

\section{Problem definition and assumption}

\subsection{Notations}

The sets, parameters, and decision variables, which are used in this research, are introduced in following sub-sections.

\subsection{Sets}

I Set of potential depot locations $i \epsilon\{1, \ldots, m\}$,

$J$ Set of customers $j \epsilon\{1, \ldots, n\}$,

$K$ Set of transportation vehicles $k \epsilon\{1, \ldots, k\}$,

\subsection{Model Parameters}

$n \quad$ Number of customers

$P \quad$ Number of depot locations

$K \quad$ Number of available vehicles

$t_{i j} \quad$ Traveling time from point $i$ to point $(i, j \in I \cup J)$

$F_{i} \quad$ The fixed cost of opening a depot at site $i$

$C_{i j} \quad$ The unit cost of transportation from point $i$ to point $j(i, j \in I U J)$

$d_{i j} \quad$ Distance among point $i$ and point $j(i, j \in I \cup J)$

$F V_{k} \quad$ The fixed cost of transportation by vehicle $k$

$D_{j} \quad$ The demand of costumer $j$

$S_{j k} \quad$ Service time of vehicle $k$ in place of costumer $j$

$W_{j k} \quad$ Waiting time of vehicle $k$ in place of costumer $j$

$D I \quad$ Imbalance between distances traveled by vehicles

$Q \quad$ Fixed loading capacity of transportation vehicles

\subsection{Decision Variables}

$X_{i j k} \quad$ It is equal to 1 , if point $j$ is immediately met after point $i$ by vehicle $k(i, j \in I \cup J, k \in K)$; 0 otherwise.

$Y_{i} \quad$ Equal to 1, if depot is located at site $i ; 0$ otherwise.

$Z_{i j} \quad$ Equal to 1, if customer $\mathrm{j}$ is served by depot $i ; 0$ otherwise.

$U_{i k}$ Auxiliary variables used in sub-tour elimination.

\subsection{The Mathematical Model}

In this section, a mathematical model of multi-objective LRP with capacitated vehicles is proposed. To formulate the problem, let $G=(V, E)$ be an undirected simple graph where $V$ is a set of nodes comprised of a subset $I$ of $P$ potential depot locations and a subset $J=V / I$ of n customers. Furthermore, the arc set E includes the pairs $e=(i, j)$, where each depot site $i \epsilon I$ has a fixed opening cost, and each customer $j$ $\epsilon J$ has a demand that must be fulfilled by a single vehicle. The unit cost of transportation is determined. A set $K$ of homogeneous vehicles with limited capacity is available. Each vehicle incurs a dependent cost when used by a depot $i$ and also navigates a single route. Each route must begin from and terminates 
at the same depot, while its total load must not exceed vehicle capacity, and no route is considered between depots. In addition, the sum of vehicles' traveling time, service time $\left(S_{j k}\right.$, $)$ and waiting time $\left(W_{j k}\right)$ must be lower than a predetermined value of $B$.

$$
\begin{aligned}
& \min \sum_{i \in I} F_{i} y_{i}+\sum_{k \in K} F V_{k} \sum_{i \in I} \sum_{j \in J} x_{i j k}+\sum_{i \in I} \sum_{j \in J} \sum_{k \in K} C_{i j} d_{i j} X_{i j k} \\
& \min D I=\max \left(\sum_{i \in I} \sum_{j \in J} d_{i j} X_{i j k}\right)-\min \left(\sum_{i \in I} \sum_{j \in J} d_{i j} X_{i j k}\right)
\end{aligned}
$$

subject to:

$$
\begin{aligned}
& \sum_{i \in I}{ }_{i} y_{i}=P \\
& \sum_{k \in K} \sum_{i \in I \cup J} x_{i j k}=1 \\
& \sum_{j \in I \cup J} x_{i j k}-\sum_{j \in I \cup J} x_{j i k}=0 \\
& \sum_{i \in I} \sum_{j \in J} x_{i j k} \leq 1 \\
& \sum_{j \in J} D_{j} \sum_{i \in I \cup J} x_{i j k} \leq Q \\
& -z_{i j}+\sum_{u \in I \cup J}\left(x_{i u k}+x_{u j k}\right) \leq 1 \\
& \sum_{i \in I \cup J} \sum_{j \in I \cup J} t_{i j} x_{i j k} \\
& +\sum_{j \in J}\left(S_{j k}+w_{j k}\right) \sum_{j \in I \cup J} x_{i j k} \leq B \\
& z_{i j} \leq y_{i} \\
& U_{g k}-U_{j k}+N x_{g j k} \leq N-1 \\
& x_{i j k}, y_{i}, z_{i j} \in\{0,1\} \\
& U_{l k} \geq 0
\end{aligned}
$$$$
\forall k \in K
$$

The objective function Eq. (1) seeks to minimize the total cost of the system consisting of depot opening cost, and fixed and variable transportation costs. The objective function (2) seeks to minimize the value of DI, in order to reduce the difference between distances traveled by vehicles. This objective function is useful to find the optimum routes of vehicles and optimum sequence to serve customers as well as having a better trade-off between two problems of facility location and vehicle routing problem. Constraint (3) ensures that the number of depot locations is equal to a predetermined value. Eq. (4) ensures that each customer is allocated to a single opened depot and be served using only one transportation vehicle. Constraint (5) gives a guarantee in that each route must begin from and end at the same depot. This means that a given vehicle departed a customer that it had served. The set of Eq. (6) ensures that there can be a maximum of one rout between two costumers. Constraint (7) is used to limit the capacity of vehicles. The set of Constraint (8) states that the demand can be assigned to the customers only in a route including both of depot and customers. Constraint (9) guarantees that the vehicles' total traveling time, service time and waiting time must be lower than a given amount of $B$. The set of Constraint (10) ensures that a depot can serve the customers when it is established and Constraint (11) guarantees the sub-tour elimination. Finally, the set of Constraints (12) to (13) are used to represent the nature of the decision variables. 


\section{Proposed Algorithms and Solution Approaches}

Since the problem of location-routing is known as an NP-hard problem (Salhi \& Nagy, 1999), metaheuristic search algorithms are utilized to solve the problem, especially in large instances. In this paper, we propose a new Multi-Objective Imperialist Competitive Algorithm (MOICA) to solve the presented multi-objective mathematical model. Furthermore, to demonstrate the efficiency of the suggested MOICA, a well-known multi-objective evolutionary algorithms, named NSGA-II (Non-dominated Sorting Genetic Algorithm II) is presented. In order to efficiently search the solution space of the given problem, several mutation and crossover strategies are defined and customized for both algorithms based on response surface methodology. Finally, a comparison study is conducted to validate the performance of the proposed MOICA with respect to two existing performance measures considering several benchmark instances. Both algorithms applied in this paper are coded using MATLAB software and run on a personal computer. In the following subsections: solution representation scheme, proposed MOICA, comparative meta-heuristic algorithms, parameter setting, comparison metrics, initialization of numerical test problems and finally comparison of meta-heuristic algorithms are presented.

\subsection{Solution Representation and Initialization}

A candidate solution must find out the allocated customers to each vehicle, the open depot centers and the sequence of customers which are served by the same vehicle, in a what each vehicle begins and terminates at the same depot. In this study, candidate solution is represented using a string of numbers containing a permutation of $n$ customers, $m$ vehicles and $d$ potential depots. These numbers are in three distinct parts in which the first two parts (first $n+m$ elements) are used to decode the solution and the final $\mathrm{m}$ part may be decoded separately. The first part ( $n$ element) in a solution indicates costumer sequence in each rout. The second part ( $m$ element) shows the customer indices to be served in each vehicle's rout. The third part ( $m$ element) determines routs to start from each depot, in which the depot serves those customers between itself and the next depot in the third part of solution representation. In such a way that the first route of depot begins by serving to the first customer after the depot. Other customers assigned to this depot are added to the current route one by one, from left to right. The current route will be also terminated whenever adding the next customer's demand will resulted in exceeding of the vehicle's capacity. Then a new route will be started to serve remaining customers allocated to this depot. It can be easily verified that the vehicle capacity constraint is not violated.

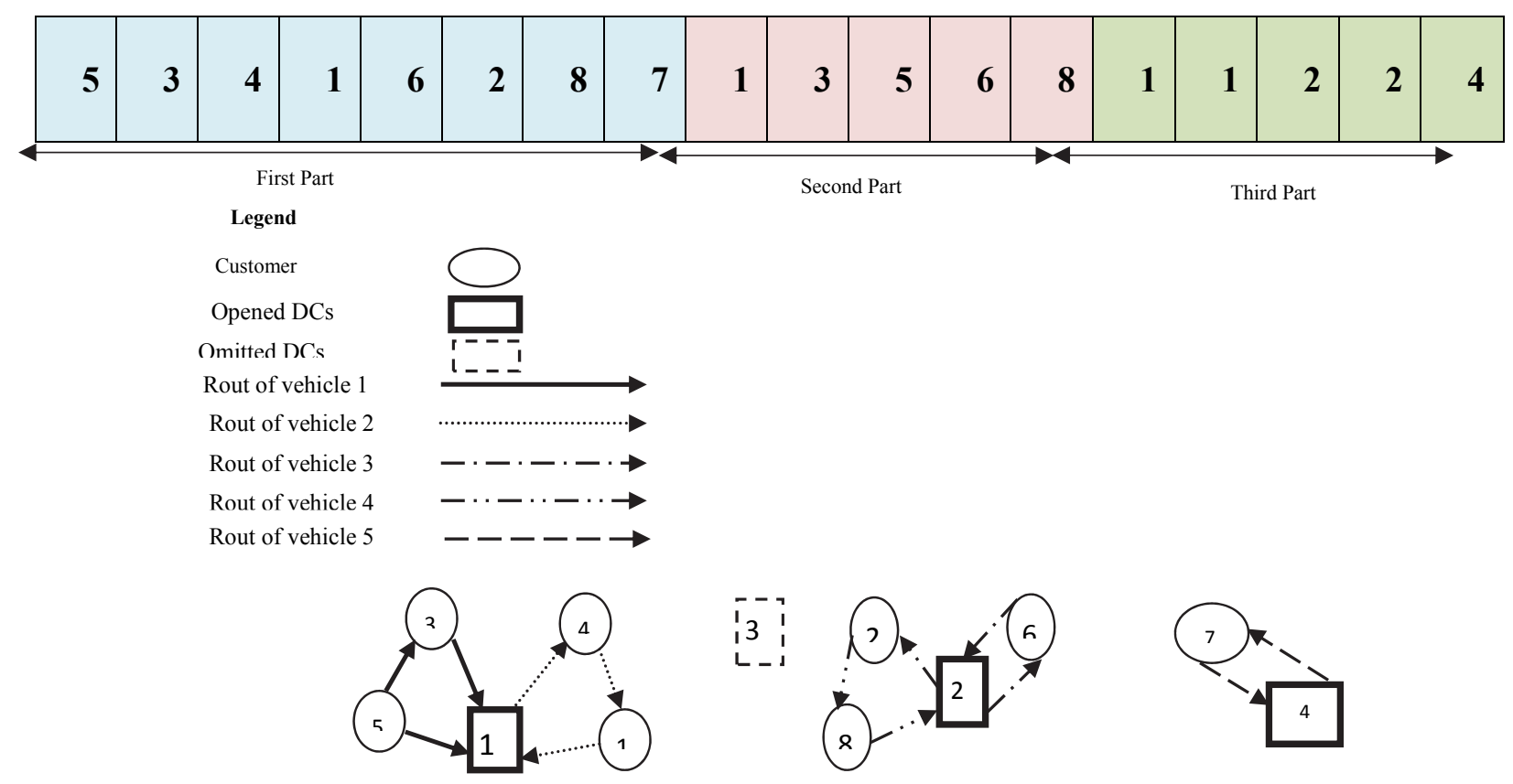

Fig. 1. Sample solution representation 
Fig. 1 gives a visual illustration of the sample solution for an example of eight costumers, five vehicles and four candidate locations for depots. The first part of the represented solution indicates that costumers must be served according to the order [5-3-4-1-6-2-7-8]. The second part of the solution exposes the costumer indices, which are served using each vehicle. Therefore, the numbers of elements in this part must be equal to the number of vehicles. The costumers, which their indices are among the values assigned to two consecutive elements of second part (ith and $(i+1)$ th elements), must be served by a single vehicle. Hence, the value of last element in second part is equal to $n$, regarding $n$ customers. Moreover, the values of second part must be sorted from smallest to largest. In this sample of represented solution, the customers with indices 1 and 2 (5th and 3 th customer) are served by vehicle 1 , the customer with indices 3 and 4 (4th and 1th customer) are served by vehicle 2, the customer with index 5 is served by vehicle 3, and the customer with indices 6 and 7 (2th and 8th customer) are served by vehicle 4 and finally the customers with index 8 ( 7 th customer) is served by vehicle 5 . In addiltion, the third part determines that vehicles 1 and 2 must start from the first depot and the second depot is the starting node for third and fourth vehicles, the third candidate depot does not host a vehicle and the forth depot is the starting node for fifth vehicle. By removing duplicate values in third part, the indices of opened depots can be easily found. This representation of solution is effective and easy to decode.

\subsection{Multi-Objective Imperialist Competitive Algorithm}

Imperialist Competitive Algorithm (ICA) is a socio-politically motivated global search algorithm that has recently been presented to solve different optimization problems (Atashpaz-Gargari \& Lucas, 2007). It is shown that this algorithm has high convergant rate and also has the ability to achieve better global solutions (Nazari-Shirkouhi et al., 2010). The main steps of this algorithm is explained in more detail in the following sub-sections.

\subsubsection{Generating initial empires}

To solve $\mathrm{N}$-dimensional optimization problem, a country is formed as an array as follows:

$$
\text { Country }=\left[p_{1}, p_{2}, p_{3}, \ldots, p_{N}\right]
$$

The cost of a country is calculated on the basis of the cost functions, shown as:

$$
\operatorname{Cos} t=f[\text { Country }]=f\left[p_{1}, p_{2}, p_{3}, \ldots, p_{N}\right]
$$

To start the optimization algorithm, an initial population size, $N_{p o p}$, is generated and then a number of most powerful countries, $N_{i m p}$, are considered as imperialists. The remaining of the population, $N_{\text {col }}\left(N_{c o l}=N_{\text {pop }}-N_{\text {imp }}\right)$, forms colonies that belong to an empire. The cost of each objective function is obtained by:

$$
\operatorname{Cos} t_{i, n}=\left|\frac{f_{i, n}^{p}-f_{i, n}^{p, b e s t}}{f_{i, \text { total }}^{p, \text { max }}-f_{i, \text { total }}^{p, \min }}\right|,
$$

where $\operatorname{Cos} t_{i, n}$ denotes the normalized value of the objective function $i$ for imperialist $n$, and $f_{i, n}^{p}$ shows the value of the objective function $i$ for imperialist $n$. The best values for the objective function $i$ at each iteration are given by $f_{i, n}^{p, b e s t}$ and the maximum and minimum values are represented as $f_{i, t o t a l}^{p, \max }$ and $f_{i, t o t a l}^{p, \text { in }}$, respectively. The summation of the normalized value for all objective functions is used to calculate the normalized cost value of each imperialist, as follows:

$$
\text { Total Cos } t_{n}=\sum_{i=1}^{r} \cos t_{i, n}
$$


In Eq. (16), $r$ is the number of objective functions. Then, the power of each imperialist is obtained and the colonies are divided among them due to imperialists'power and authority as follows:

$$
p_{n}=\left|\frac{\text { Total } \operatorname{Cos} t_{n}}{\sum_{i=1}^{N_{\text {imp }}} \text { Total } \operatorname{Cos} t_{i}}\right| .
$$

Finally, the initial number of colonies of an empire is obtained using th following equation:

$$
N C_{n}=\operatorname{round}\left\{p_{n}, N_{c o l}\right\} \text {, }
$$

where, $N C_{n}$ represents the initial number of colonies of $n$th imperialist, and $N_{c o l}$ is the number of all colonies. We randomly choose $N_{c o l}$ of the colonies and assign them to the imperialists. These colonies along with relevant imperialist will form $n$th empire in a way that powerful empires have greater number of colonies and weaker ones have less number.

\subsubsection{Total Power of an Empire}

The total power of an empire is calculated by the sum of imperialist's power and a percentage of mean power of its colonies which is given in Eq. (19). Then, the imperialistic competition starts and weak empires will be omitted from the competition

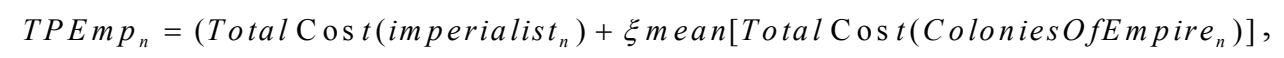

where $T P E m p_{n}$ is the total power of the $n$-th empire and $\xi(z e t a)$ is a positive small number, which is advised to be less than 1 to increase the role of the colonies in calculating the total power of an empire.

\subsubsection{Assimilation}

With the absorption policy, colonies are divided among their relevant imperialists based on the different socio-political axis. The movement direction is a vector from colony toward imperialist, as shown in Fig. 2.

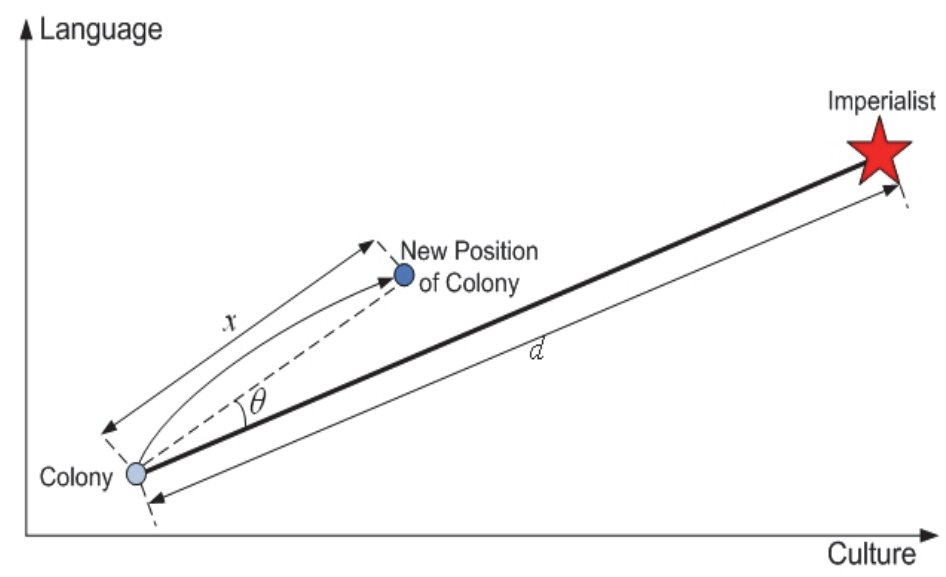

Fig. 1. Moving colonies toward the imperialists angle $\theta$

\subsubsection{Crossover Policy}

In this step, colonies share their information between themselves using crossover operators. The percentage of shared information via crossover operators is shown by $p$-Crossover.

\subsubsection{Revolution}

Meanwhile, a number of colonies are selected using a random selection mechanism and replaced with an equal number of new generated ones. 


\subsubsection{Exchanging Positions of a Colony and the Imperialist}

When a colony becomes more powerful than $i$ ts imperialist, their position will be changed.

\subsubsection{Uniting Similar Empires}

When two imperialists become too closed together in a way that the distance between them becomes less than threshold distance, they will form a new empire.

\subsubsection{Imperialistic Competition}

An imperialistic competition begins between all empires to possess some of the weakest colonies of the weakest empire based on their possession probability by Eq. (20).

$$
N T P E m p_{n}=\max \left\{T P E m p_{i}\right\}-T P E m p_{n}
$$

In which NTPEmp $p_{n}$ is the total power of the $n$-th empire and TPEmp $p_{n}$ is its normalized total power. The normalized power or the possession probability of each empire can be stated by:

$$
P_{P_{n}}=\left|\frac{N T P E m p_{n}}{\mid \sum_{i=1}^{N_{i m p}} T N T P E m p_{i}}\right|
$$

Then, the mentioned colony is assigned to one of the empires using roulette wheel method. Firstly, the vector $P$ is formed to divide the weakest colonies between empires as follows:

$$
P=\left[p_{P_{1}}, p_{P_{2}}, p_{P_{3}}, \ldots, p_{P_{N}}\right]
$$

Then the vector $R$ with the same size as $P$ is obtained, which its elements are random numbers generated using uniform distribution function between 0 and 1 .

$$
R=\left[r_{1}, r_{2}, r_{3}, \ldots, r_{N_{i n p}}\right]
$$

Finally, vector $D$ is formed using subtracting vector $R$ from $P$, as follows:

$$
D=P-R=\left[D_{1}, D_{2}, D_{3}, \ldots, D_{N_{\text {inp }}}\right]=\left[p_{P_{1}}-r_{1}, p_{P_{2}}-r_{2}, p_{P_{3}}, \ldots, p_{p_{N_{\text {imp }}}}-r_{N_{\text {imp }}}\right]
$$

Considering vector $D$, the index of largest element determines the empire that will possess the colony (colonies).

\subsubsection{Eliminating the Powerless Empires}

Pursuing the imperialistic competition process, weak empires will be eliminated. This strategy will gradually increase the power of more strong empires and decrease the power of weaker ones.

\subsubsection{Stopping Criteria}

There are different criteria to stop an algorithm such as using a number of maximum iterations of the algorithm, predefined CPU time, or remaining only one empire during the competition process. In this paper, the proposed algorithm will be stoped when the countries converge to the global minimum of the cost and only one empire remains.

\subsection{Comparative Meta-Heuristic (NSGA II)}

Here, we endeavor to evaluate and demonstrate the efficiency of our proposed MOICA by comparison with a famous multi-objective evolutionary algorithms, named NSGA-II (non-dominated sorting genetic algorithm II), in terms of two comparison metrics which will be introduced in section 4.4. NSGA-II introduced by (Deb et al., 2000; Deb \& Pratap, 2002), is an extension of the Genetic 
Algorithm for obtaining Pareto-optimal front for multi-objective optimization problems. NSGA-II is a revised version of NSGA (Srinivas \& Deb, 1994). In the proposed NSGA-II, crossover and mutation operators are used to generate new solutions and then the following fundamental issues are employed:

\subsubsection{Non-Dominated Sorting}

Non-dominated sorting seeks to divide candidate solution in to small groups in a way that there is no one solution superior to the others for all objective functions. Then the best of them will be selected.

\subsubsection{Crowding Distance}

Crowding distance sorting removes a series of subsets from the small groups created by the nondominated technique, so that these subsets may from the other ones. The crowding distance used in the NSGA-II is calculated as follows:

$$
C D_{i}=\sum_{r=1}^{n} \frac{f_{r, i+1}^{p}-f_{r, i-1}^{p}}{f_{r, \text { total }}^{p+\max }-f_{r, \text { total }}^{p+\min }}
$$

In which, $n$ is the number of objective functions, $f_{r, i+1}^{p}$ is the $r$ th objective function of the $i+1$ th solution and $f_{r, i-1}^{p}$ is the $r$ th objective function of the $i$ - 1 th solution. Also, $f_{r, \text { total }}^{p, \max }$ and $f_{r, \text { total }}^{p, \min }$ denote the maximum and minimum values of the $r$ th objective function.

\subsubsection{Selection and Recombination}

The selection is performed by tournament selection with crowed comparison-operator. This procedure combines the current generation population and the offspring population in order to select individuals for new generation.

\subsubsection{Parameter tuning}

The efficiency of an algorithm is greatly dependent on its parameters. To tune the parameters of the algorithms that ensure the high quality solutions, we notice two different sizes of LRP problems, namely small sizes and large sizes. In order to find out the values of parameters, we used response surface methodology (RSM), that can result in continuous parameter setting compared to factorial design ( Montgomery, 1997; Zandieh et al., 2009). First, we have to find out factors which are statistically significant for each algorithm in the cases of makespan and elapsed time. Each factor is noticed at two levels, which can be coded in a way that the high value becomes "1" and the low value becomes "-1". Coded variable can be stated as below:

$$
x_{i}=\frac{r_{i}-\left(\frac{h+l}{2}\right)}{\left(\frac{h-l}{2}\right)}
$$

In which, $x_{i}$ and $r_{i}$ represent coded variable and natural variable, respectively. Also, $h$ and $l$ show high level and low level of factor. Moreover, we need an index to compare different combinations of parameters. Since the LRP model presented in this paper is a multi-objective, so we should employ a unique index such as quality index to compare the parameters. In this method, all of the Pareto solutions obtained by different parameter combinations are considered together, and then non-dominance operation is done for all of them concurrently. The percentage of the Pareto solution related to each parameter combination is considered as its quality index. In the presented paper, the NFC is set 30000 for small-sized problems and 100000 for the large-sized problems. Considered parameters and their levels for small and large sized problems in the MOICA algorithm are shown in Table 1. In addition, the tuned value parameters for small and large sized problems of the proposed MOICA are shown in Table 2. Furthermore, for NSGA-II, the initial population sizes are set 200 for small-sized and 300 for large-sized problems. The mutation rate and the crossover rate is set 0.2 and 0.8 , respectively. The 
stopping criterion is considered to be the NFC and is set at the value of 30000 for large-sized and 100000 for small-sized problems, respectively.

Table 1

Parameters and their levels of the MOICA for small and large sized problems

\begin{tabular}{|c|c|c|c|c|c|c|}
\hline \multirow[b]{3}{*}{ Factors } & \multicolumn{6}{|c|}{ Coded level } \\
\hline & \multicolumn{2}{|c|}{-1} & \multicolumn{2}{|c|}{0} & \multicolumn{2}{|c|}{+1} \\
\hline & $\begin{array}{c}\text { Small sized } \\
\text { problems }\end{array}$ & $\begin{array}{c}\text { Large sized } \\
\text { problems }\end{array}$ & $\begin{array}{c}\text { Small sized } \\
\text { problems }\end{array}$ & $\begin{array}{l}\text { Large sized } \\
\text { problems }\end{array}$ & $\begin{array}{c}\text { Small sized } \\
\text { problems }\end{array}$ & $\begin{array}{l}\text { Large sized } \\
\text { problems }\end{array}$ \\
\hline n-Pop & 100 & 150 & 150 & 225 & 200 & 300 \\
\hline N-imp & 4 & 8 & 6 & 10 & 8 & 12 \\
\hline $\mathrm{P}_{\mathrm{A}}$ & 0.3 & 0.4 & 0.5 & 0.6 & 0.7 & 0.8 \\
\hline $\mathrm{P}_{\mathrm{C}}$ & 0.2 & 0.3 & 0.4 & 0.5 & 0.6 & 0.7 \\
\hline$P_{R}$ & 0.1 & 0.2 & 0.2 & 0.3 & 0.3 & 0.4 \\
\hline$\xi$ & 0.1 & 0.1 & 0.15 & 0.15 & 0.2 & 0.2 \\
\hline$\beta$ & 1 & 1 & 2 & 2 & 3 & 3 \\
\hline
\end{tabular}

Table 2

Tuned value parameters of the MOICA

\begin{tabular}{|c|c|c|c|c|}
\hline \multirow[b]{3}{*}{ Factors } & \multicolumn{4}{|c|}{ Coded level } \\
\hline & \multicolumn{2}{|c|}{ Optimal coded value } & \multicolumn{2}{|c|}{ Optimal real value } \\
\hline & $\begin{array}{l}\text { Small sized } \\
\text { problems }\end{array}$ & $\begin{array}{l}\text { Large sized } \\
\text { problems }\end{array}$ & $\begin{array}{c}\text { Small sized } \\
\text { problems }\end{array}$ & Large sized problems \\
\hline n-Pop & 0.85 & 1 & 193 & 300 \\
\hline $\mathrm{N}$-imp & -0.2 & -1 & 5 & 8 \\
\hline $\mathrm{P}_{\mathrm{A}}$ & 0.18 & 0.2 & 0.54 & 0.64 \\
\hline $\mathrm{P}_{\mathrm{C}}$ & 1 & 0.5 & 0.6 & 0.6 \\
\hline$P_{R}$ & -0.8 & 0.19 & 0.12 & 0.32 \\
\hline$\xi$ & 0.9 & -0.5 & 0.195 & 0.125 \\
\hline$\beta$ & -0.2 & 0.15 & 1.8 & 2.15 \\
\hline
\end{tabular}

\subsection{Comparison Metric}

In order to validate the proposed MOICA, two well-known comparison metrics which are appropriate for multi-objective algorithms are considered.

\subsubsection{Quality Metric (QM)}

Using this method, all of the non-dominated solutions acquired by the algorithms are considered together and the percentage of the Pareto solution for each algorithm is then calculated. Considering this metric, the solution with a higher value is known as a better solution (Moradi et al., 2011).

\subsubsection{Mean ideal distance (MID)}

MID denotes the distance between the best and Pareto solutions, which is calculated as Eq. (27). In contrast to the QM, in this metric the solution with a lower value of the MID is known as a better solution.

$$
M I D=\frac{\sum_{i=1}^{n} \sqrt{\left(\frac{f_{1 i}-f_{1}^{\text {best }}}{f_{1, \text { total }}^{\max }-f_{1, \text { total }}^{\min }}\right)^{2}+\left(\frac{f_{2 i}-f_{2}^{\text {best }}}{f_{2, \text { total }}^{\max }-f_{2, \text { total }}^{\min }}\right)^{2}}}{n}
$$

In which, $n$ is the number of non-dominated solutions, $f_{1, \text { total }}^{\max }$ is the maximum value of $i$-th fitness function among all non-dominated solutions obtained by the algorithms, $f_{1, \text { total }}^{\min }$ is the minimum value of $i$-th fitness functions among all non-dominated solutions obtained by the algorithms and $f_{1}^{\text {best }}$ is the best solution of $i$-th fitness function. 


\subsection{Generating numerical test problems}

It is known that meta-heuristics are greatly sensitive to their initial solutions and it plays an important role in reaching better solutions. Hence, to verify the efficiency of the proposed model and algorithms, numerical test problems are generated and different size of test problems is also taken in to account to conduct computational experiments and compare the results of each algorithm. First, Table 3 shows the parameters which are randomly generated from the uniformly distributed intervals.

Table 3

Corresponding parameters

\begin{tabular}{llll}
\hline Parameters & Corresponding value & Parameters & Corresponding value \\
\hline $\mathrm{d}_{\mathrm{ij}}$ & Uniform $\sim(10,20)$ & $\mathrm{D}_{\mathrm{j}}$ & Uniform $\sim(100,800)$ \\
$\mathrm{C}_{\mathrm{ij}}$ & Uniform $\sim(50,70)$ & $\mathrm{S}_{\mathrm{jk}}$ & Uniform $\sim(12,30)$ \\
$\mathrm{t}_{\mathrm{ij}}$ & Uniform $\sim(30,100)$ & $\mathrm{W}_{\mathrm{jk}}$ & Uniform $\sim(8,35)$ \\
$\mathrm{F}_{\mathrm{i}}$ & Uniform $\sim(2000,8000)$ & $\mathrm{Q}$ & Uniform $\sim(400,900)$ \\
$\mathrm{FV}_{\mathrm{k}}$ & Uniform $\sim(200,900)$ & $\mathrm{B}$ & Uniform $\sim(1000,8000)$ \\
\hline
\end{tabular}

After adjustment the parameters of the problems, several variable numbers of potential depots, regarding the number of costumers, are considered for each test problem; which are given in Table 4. Finally, 53 problem classes are generated to conduct computational experiments. The name of each test problems can be defined using the number of costumers, the sign of \#, and the number of candidate depots. For example, a problem with 70 costumers and 12 potential depots is defined as $70 \# 12$.

Table 4

Numbers of potential depots for each test problem.

\begin{tabular}{llllllllll}
\hline \multicolumn{10}{c}{ Number of costumers } \\
\hline & 10 & 15 & 20 & 25 & 30 & 40 & 50 & 70 & 100 \\
\hline Number of potential depots & 3 to 4 & 3 to 5 & 3 to 6 & 3 to 6 & 3 to 8 & 3 to 10 & 3 to 12 & 3 to 16 & 3 to 18 \\
\hline
\end{tabular}

\subsection{Comparison of meta-heuristic algorithms}

In order to compare the numerical solutions generated by the proposed MOICA, all the 53 given problem classes are solved via two suggested algorithms for four times and the best obtained solutions are considered as the final output of each algorithm.

\section{Table 5}

Comparison results between MOICA and NSGA-II in terms of QM and MID for small size problems

\begin{tabular}{|c|c|c|c|c|}
\hline \multirow{2}{*}{ Problem No. } & \multicolumn{2}{|c|}{ Quality Metric (QM) } & \multicolumn{2}{|c|}{ Mean Ideal Distance (MID) } \\
\hline & NSGA-II & MOICA & NSGA-II & MOICA \\
\hline $10 \# 3$ & 0.235 & 0.765 & 0.633 & 0.518 \\
\hline $10 \# 4$ & 0.105 & 0.895 & 0.704 & 0.581 \\
\hline $15 \# 3$ & 0.250 & 0.750 & 0.873 & 0.242 \\
\hline $15 \# 4$ & 0 & 1 & 0.712 & 0.348 \\
\hline $15 \# 5$ & 0 & 1 & 0.339 & 0.230 \\
\hline $20 \# 3$ & 0 & 1 & 0.776 & 0.523 \\
\hline $20 \# 4$ & 0.235 & 0.765 & 0.440 & 0.399 \\
\hline $20 \# 5$ & 0.434 & 0.565 & 0.518 & 0.538 \\
\hline $20 \# 6$ & 0.347 & 0.434 & 0.575 & 0.621 \\
\hline $25 \# 3$ & 0.100 & 0.900 & 0.601 & 0.247 \\
\hline $25 \# 4$ & 0.272 & 0.727 & 0.663 & 0.718 \\
\hline $25 \# 5$ & 0.292 & 0.708 & 0.536 & 0.511 \\
\hline $25 \# 6$ & 0.190 & 0.809 & 0.482 & 0.287 \\
\hline $30 \# 3$ & 0.167 & 0.750 & 0.697 & 0.632 \\
\hline $30 \# 4$ & 0.059 & 0.647 & 0.762 & 0.485 \\
\hline $30 \# 5$ & 0 & 1 & 0.781 & 0.500 \\
\hline $30 \# 6$ & 0 & 1 & 0.297 & 0.379 \\
\hline $30 \# 7$ & 0.118 & 0.882 & 0.479 & 0.276 \\
\hline $30 \# 8$ & 0 & 1 & 0.579 & 0.554 \\
\hline
\end{tabular}

Then, the performance of the MOICA is compared with the NSGA-II regarding two proposed comparison metrics. The comparison results for small, medium and large-sized problems are illustrated 
in Tables 5 to 8. Based on the comparison results, the QM metric indices in the MOICA have a higher value and the MID metric indices have a lower value than NSGA-II for all of the test problems. Hence, according to two comparison metrics, the proposed MOICA is superior to NSGA-II and also it shows considerably better performance in large-size problems (Table 8) compared to small-size problems (Table 5). In addition, dispersions of non-dominated solutions obtained via MOICA and NSGA-II with respect to the values of both objective functions, for problem with 70 costumers and 12 potential depots, are considered simultaneously in Fig. 4. As it seen, the proposed MOICA is much superior to NSGAII and improving it's effectiveness can be obviously observed along with the increase in problem size, that leads to more complexity.

Table 1

Comparison results between MOICA and NSGA-II in terms of QM and MID for medium size problems

\begin{tabular}{ccccc}
\hline \multirow{2}{*}{ Problem No. } & \multicolumn{2}{c}{ Quality Metric (QM) } & \multicolumn{2}{c}{ Mean Ideal Distance (MID) } \\
\cline { 2 - 5 } & NSGA-II & MOICA & NSGA-II & MOICA \\
\hline $40 \# 3$ & 0.071 & 0.928 & 0.707 & 0.430 \\
$40 \# 4$ & 0.3634 & 0.364 & 0.609 & 0.452 \\
$40 \# 5$ & 0.357 & 0.642 & 0.554 & 0.377 \\
$40 \# 6$ & 0.318 & 0.500 & 0.664 & 0.408 \\
$40 \# 7$ & 0.370 & 0.630 & 0.731 & 0.563 \\
$40 \# 8$ & 0.0416 & 0.958 & 0.430 & 0.322 \\
$40 \# 9$ & 0.240 & 0.760 & 0.506 & 0.431 \\
$40 \# 10$ & 0.111 & 0.778 & 0.692 & 0.360 \\
\hline
\end{tabular}

Table 2

Comparison results between MOICA and NSGA-II in terms of QM and MID for medium size problems

\begin{tabular}{|c|c|c|c|c|}
\hline \multirow[t]{2}{*}{ Problem No. } & \multicolumn{2}{|c|}{ Quality Metric (QM) } & \multicolumn{2}{|c|}{ Mean Ideal Distance (MID) } \\
\hline & NSGA-II & MOICA & NSGA-II & MOICA \\
\hline $50 \# 3$ & 0.434 & 0.478 & 0.641 & 0.658 \\
\hline $50 \# 4$ & 0.105 & 0.895 & 0.526 & 0.492 \\
\hline $50 \# 5$ & 0.238 & 0.762 & 0.603 & 0.443 \\
\hline $50 \# 6$ & 0 & 1 & 0.554 & 0.482 \\
\hline $50 \# 7$ & 0 & 1 & 0.490 & 0.383 \\
\hline $50 \# 8$ & 0 & 1 & 0.457 & 0.281 \\
\hline $50 \# 9$ & 0 & 1 & 0.504 & 0.369 \\
\hline $50 \# 10$ & 0 & 0.892 & 0.702 & 0.373 \\
\hline $50 \# 11$ & 0.160 & 0.840 & 0.585 & 0.640 \\
\hline $50 \# 12$ & 0 & 1 & 0.680 & 0.230 \\
\hline
\end{tabular}

Table 8

Comparison results between MOICA and NSGA-II in terms of QM and MID for large size problems

Problem No.

\section{Quality Metric (QM)}

\begin{tabular}{ccccc} 
Problem No. & NSGA-II & MOICA & NSGA-II & MOICA \\
\cline { 2 - 5 } & 0 & 1 & 0.492 & 0.125 \\
$70 \# 3$ & 0 & 1 & 0.519 & 0.250 \\
$70 \# 4$ & 0 & 1 & 0.959 & 0.365 \\
$70 \# 5$ & 0 & 1 & 0.672 & 0.174 \\
$70 \# 6$ & 0.200 & 0.800 & 0.692 & 0.336 \\
$70 \# 7$ & 0 & 1 & 0.509 & 0.364 \\
$70 \# 8$ & 0 & 0.924 & 0.643 & 0.257 \\
$70 \# 9$ & 0 & 1 & 0.696 & 0.222 \\
$70 \# 10$ & 0 & 1 & 0.275 & 0.250 \\
$70 \# 11$ & 0 & 1 & 0.643 & 0.127 \\
$70 \# 12$ & 0 & 1 & 0.518 & 0.220 \\
$70 \# 13$ & 0.352 & 0.647 & 0.491 & 0.459 \\
$70 \# 14$ & 0.273 & 0.727 & 0.456 & 0.256 \\
$70 \# 15$ & 0 & 1 & 0.847 & 0.210 \\
\hline
\end{tabular}
(MID)

Mean Ideal Distance 


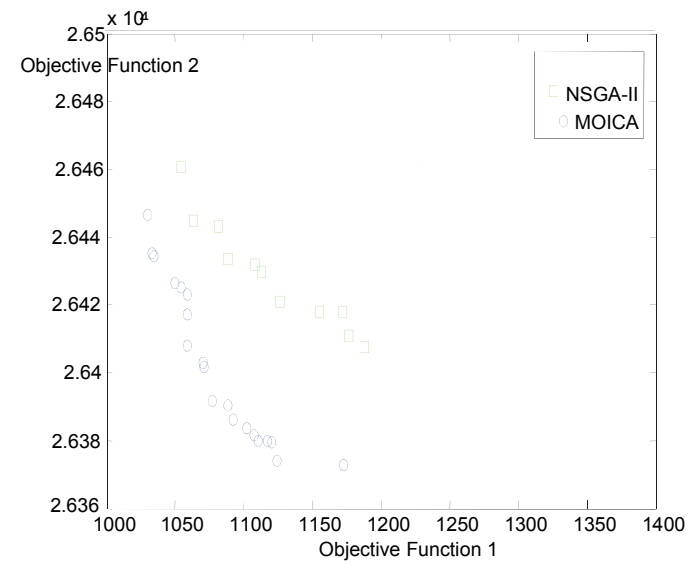

Fig. 4. Dispersion of non-dominated solutions obtained by MOICA and NSGAII

\section{Conclusion}

In this paper a novel multi-objective imperialist competitive algorithm (MOICA) has been presented to solve a multi-objective LRP with capacitated and homogeneous vehicle fleet. The proposed model considered vehicle's traveling distance, service time and waiting time while it guaranteed that the sum of these parameters be lower than a predetermined value. The monetory objective function was to minimize the total cost of the system. The non-monetary objective function minimizes the difference between vehicle's traveled distance; which is useful to find the optimum routes of vehicles and optimum sequence to serve customers. The problem is solved via MOICA and its performance has been validated via comparing with NSGA II. To this order, after adjustment several crossover and mutation strategies for each algorithm based on response surface methodology, a comparison study was conducted. The associated results in terms of two well-known comparison metrics on several benchmark instances demonstrated that the proposed MOICA outperforms the NSGA II and also it shows considerably better performance in large-size problems. To continue the current research, some other related transportation issues can be considered, such as availability of facilities (time window) and model with pickup and delivery demands. Also, using fuzzy and probabilistic parameters and hybridizing the proposed MOICA with new local search procedures can be valuable research subjects.

\section{References}

Ahn, J., de Weck, O., Geng, Y., \& Klabjan, D. (2012). Column generation based heuristics for a generalized location routing problem with profits arising in space exploration. European Journal of Operational Research, 223(1), 47-59.

Atashpaz-Gargari, E., \& Lucas, C. (2007). Imperialist competitive algorithm: an algorithm for optimization inspired by imperialistic competition. Paper presented at the Evolutionary computation, 2007. CEC 2007. IEEE Congress on.

Balakrishnan, A., Ward, J. E., \& Wong, R. T. (1987). Integrated facility location and vehicle routing models: Recent work and future prospects. American Journal of Mathematical and Management Sciences, 7(1-2), 3561.

Boventer, E. (1961). The relationship between transportation costs and location rent in transportation problems. Journal of Regional Science, 3(2), 27-40.

C Montgomery, D. (1997). Montgomery Design and Analysis of Experiments.

Caballero, R., González, M., Guerrero, F. M., Molina, J., \& Paralera, C. (2007). Solving a multiobjective location routing problem with a metaheuristic based on tabu search. Application to a real case in Andalusia. European Journal of Operational Research, 177(3), 1751-1763.

Çetiner, S., Sepil, C., \& Süral, H. (2010). Hubbing and routing in postal delivery systems. Annals of Operations Research, 181(1), 109-124.

Christofides, N., \& Eilon, S. (1969). An algorithm for the vehicle-dispatching problem. Or, 309-318. 
Deb, K., Agrawal, S., Pratap, A., \& Meyarivan, T. (2000). A fast elitist non-dominated sorting genetic algorithm for multi-objective optimization: NSGA-II. Lecture notes in computer science, 1917, 849-858.

Deb, K., \& Pratap, A. (2002). A fast elitist non-dominated sorting genetic algorithm for multi-objective optimization: NSGA-II.

Diabat, A. (2014). Hybrid algorithm for a vendor managed inventory system in a two-echelon supply chain. European Journal of Operational Research, 238(1), 114-121.

Drexl, M., \& Schneider, M. (2015). A survey of variants and extensions of the location-routing problem. European Journal of Operational Research, 241(2), 283-308. doi: 10.1016/j.ejor.2014.08.030

Fakhrzada, M., \& Esfahanib, A. S. (2013). Modeling the time windows vehicle routing problem in cross-docking strategy using two meta-heuristic algorithms. International Journal of Engineering-Transactions A: Basics, 27(7), 1113.

Goścień, R., Walkowiak, K., \& Klinkowski, M. (2015). Tabu search algorithm for routing, modulation and spectrum allocation in elastic optical network with anycast and unicast traffic. Computer Networks, 79, 148165. doi: 10.1016/j.comnet.2014.12.004

Govindan, K., Jafarian, A., Khodaverdi, R., \& Devika, K. (2014). Two-echelon multiple-vehicle locationrouting problem with time windows for optimization of sustainable supply chain network of perishable food. International Journal of Production Economics, 152, 9-28. doi: 10.1016/j.ijpe.2013.12.028

Gu, J., Goetschalckx, M., \& McGinnis, L. F. (2007). Research on warehouse operation: A comprehensive review. European Journal of Operational Research, 177(1), 1-21. doi: 10.1016/j.ejor.2006.02.025

Jula, A., Othman, Z., \& Sundararajan, E. (2015). Imperialist competitive algorithm with PROCLUS classifier for service time optimization in cloud computing service composition. Expert Systems with Applications, 42(1), 135-145. doi: 10.1016/j.eswa.2014.07.043

Karakatič, S., \& Podgorelec, V. (2015). A survey of genetic algorithms for solving multi depot vehicle routing problem. Applied Soft Computing, 27, 519-532. doi: 10.1016/j.asoc.2014.11.005

Koç, Ç., Bektaş, T., Jabali, O., \& Laporte, G. (2015). A hybrid evolutionary algorithm for heterogeneous fleet vehicle routing problems with time windows. Computers \& Operations Research, 64, 11-27. doi: 10.1016/j.cor.2015.05.004

Kopfer, H., Schwardt, M., \& Dethloff, J. (2005). Solving a continuous location-routing problem by use of a selforganizing map. International Journal of Physical Distribution \& Logistics Management, 35(6), 390-408. doi: $10.1108 / 09600030510611639$

Maranzana, F. (1964). On the location of supply points to minimize transport costs. OR, 261-270.

Marinakis, Y., Iordanidou, G.-R., \& Marinaki, M. (2013). Particle Swarm Optimization for the Vehicle Routing Problem with Stochastic Demands. Applied Soft Computing, 13(4), 1693-1704. doi: 10.1016/j.asoc.2013.01.007

Martínez-Salazar, I. A., Molina, J., Ángel-Bello, F., Gómez, T., \& Caballero, R. (2014). Solving a bi-objective Transportation Location Routing Problem by metaheuristic algorithms. European Journal of Operational Research, 234(1), 25-36. doi: 10.1016/j.ejor.2013.09.008

Megiddo, N., \& Supowit, K. J. (1984). On the complexity of some common geometric location problems. SIAM journal on computing, 13(1), 182-196.

Min, H., Jayaraman, V., \& Srivastava, R. (1998). Combined location-routing problems: A synthesis and future research directions. European journal of operational research, 108(1), 1-15.

Mohammadi-Ivatloo, B., Rabiee, A., Soroudi, A., \& Ehsan, M. (2012). Imperialist competitive algorithm for solving non-convex dynamic economic power dispatch. Energy, 44(1), 228-240.

Mohammadkhanloo, M., \& Bashiri, M. (2013). A clustering based location-allocation problem considering transportation costs and statistical properties (research note). International Journal of EngineeringTransactions C: Aspects, 26(6), 597.

Moradi, H., Zandieh, M., \& Mahdavi, I. (2011). Non-dominated ranked genetic algorithm for a multi-objective mixed-model assembly line sequencing problem. International Journal of Production Research, 49(12), 3479-3499.

Nagy, G., \& Salhi, S. (2007). Location-routing: Issues, models and methods. European Journal of Operational Research, 177(2), 649-672. doi: 10.1016/j.ejor.2006.04.004

Nazari-Shirkouhi, S., Eivazy, H., Ghodsi, R., Rezaie, K., \& Atashpaz-Gargari, E. (2010). Solving the integrated product mix-outsourcing problem using the Imperialist Competitive Algorithm. Expert Systems with Applications, 37(12), 7615-7626. doi: 10.1016/j.eswa.2010.04.081

Norouzi, N., Sadegh-Amalnick, M., \& Alinaghiyan, M. (2015). Evaluating of the particle swarm optimization in a periodic vehicle routing problem. Measurement, 62, 162-169. doi: 10.1016/j.measurement.2014.10.024 
Or, I., \& Pierskalla, W. P. (1979). A transportation location-allocation model for regional blood banking. AIIE transactions, 11(2), 86-95.

Prodhon, C. (2011). A hybrid evolutionary algorithm for the periodic location-routing problem. European Journal of Operational Research, 210(2), 204-212. doi: 10.1016/j.ejor.2010.09.021

Prodhon, C., \& Prins, C. (2014). A survey of recent research on location-routing problems. European Journal of Operational Research, 238(1), 1-17. doi: 10.1016/j.ejor.2014.01.005

Roozbeh Nia, A., Hemmati Far, M., \& Niaki, S. T. A. (2015). A hybrid genetic and imperialist competitive algorithm for green vendor managed inventory of multi-item multi-constraint EOQ model under shortage. Applied Soft Computing, 30, 353-364. doi: 10.1016/j.asoc.2015.02.004

Salhi, S., \& Nagy, G. (1999). Consistency and robustness in location-routing. Studies in Locational Analysis(13), 3-19.

Salhi, S., \& Rand, G. K. (1989). The effect of ignoring routes when locating depots. European journal of operational research, 39(2), 150-156.

Schwardt, M., \& Fischer, K. (2008). Combined location-routing problems-a neural network approach. Annals of Operations Research, 167(1), 253-269. doi: 10.1007/s10479-008-0377-3

Setak, M., Jalili Bolhassani, S., \& Karimi, H. (2014). A node-based mathematical model towards the location routing problem with intermediate replenishment facilities under capacity constraint. International Journal of Engineering, 27(6), 911-920.

Shiripour, S., Mahdavi, I., Amiri-Aref, M., Mohammadnia-Otaghsara, M., \& Mahdavi-Amiri, N. (2012). Multifacility location problems in the presence of a probabilistic line barrier: a mixed integer quadratic programming model. International Journal of Production Research, 50(15), 3988-4008. doi: $10.1080 / 00207543.2011 .579639$

Sim, K. M., \& Sun, W. H. (2003). Ant colony optimization for routing and load-balancing: survey and new directions. Systems, Man and Cybernetics, Part A: Systems and Humans, IEEE Transactions on, 33(5), 560572.

Srinivas, N., \& Deb, K. (1994). Muiltiobjective optimization using nondominated sorting in genetic algorithms. Evolutionary computation, 2(3), 221-248.

Tavakkoli-Moghaddam, R., Makui, A., \& Mazloomi, Z. (2010). A new integrated mathematical model for a biobjective multi-depot location-routing problem solved by a multi-objective scatter search algorithm. Journal of Manufacturing Systems, 29(2-3), 111-119. doi: 10.1016/j.jmsy.2010.11.005

Ting, C.-J., \& Chen, C.-H. (2013). A multiple ant colony optimization algorithm for the capacitated location routing problem. International Journal of Production Economics, 141(1), 34-44.

Wang, H., Du, L., \& Ma, S. (2014). Multi-objective open location-routing model with split delivery for optimized relief distribution in post-earthquake. Transportation Research Part E: Logistics and Transportation Review, 69, 160-179. doi: http://dx.doi.org/10.1016/j.tre.2014.06.006

Wasner, M., \& Zäpfel, G. (2004). An integrated multi-depot hub-location vehicle routing model for network planning of parcel service. International Journal of Production Economics, 90(3), 403-419.

Watson-Gandy, C., \& Dohrn, P. (1973). Depot location with van salesmen - a practical approach. Omega, 1(3), 321-329.

Webb, M. (1968). Cost functions in the location of depots for multiple-delivery journeys. OR, 311-320.

Yu, V. F., \& Lin, S.-Y. (2015). A simulated annealing heuristic for the open location-routing problem. Computers \& Operations Research, 62, 184-196. doi: 10.1016/j.cor.2014.10.009

Zandieh, M., Dorri, B., \& Khamseh, A. (2009). Robust metaheuristics for group scheduling with sequencedependent setup times in hybrid flexible flow shops. The International Journal of Advanced Manufacturing Technology, 43(7-8), 767.

Zarandi, M. H. F., Hemmati, A., \& Davari, S. (2011). The multi-depot capacitated location-routing problem with fuzzy travel times. Expert Systems with Applications, 38(8), 10075-10084.

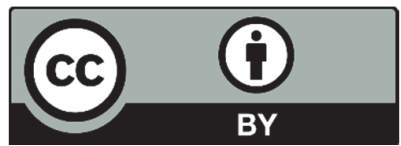

(C) 2019 by the authors; licensee Growing Science, Canada. This is an open access article distributed under the terms and conditions of the Creative Commons Attribution (CC-BY) license (http://creativecommons.org/licenses/by/4.0/). 